\title{
Efficient and Facile Synthesis of Chromenopyrano[2,3-b] pyridine Derivatives Catalyzed by Sodium Carbonate
}

\author{
Raziyeh Keshavarz, Mahnaz Farahi ${ }^{\star}$ and Bahador Karami \\ Department of Chemistry, Yasouj University, P. O. Box 353, Yasouj 75918-74831, Iran \\ *Corresponding author: E-mail: farahimb@yu.ac.ir
}

Received: 07-09-2020

\begin{abstract}
In this research, a number of new and known chromenopyrano[2,3- $b$ ]pyridine derivatives have been prepared. Initially, according to the reported procedure, pyrano[2,3-c]chromene derivatives were synthesized by the reaction between 4-hydroxycoumarin, aromatic aldehydes and malononitrile using silica sodium carbonate (SSC) as the catalyst. Next, the prepared pyrano[2,3-c]chromenes were reacted by dimethyl acetylenedicarboxylate (DMAD) or cyclohexanone in the presence of sodium carbonate to produce chromenopyrano[2,3-b]pyridine derivatives. The presented protocol avoids the use of expensive catalysts and gives useful potentially bioactive heterocycles in excellent to high yields.
\end{abstract}

Keywords: Chromenopyrano[2,3-b]pyridine; pyrano[2,3-c]chromene, dimethyl acetylenedicarboxylate, cyclohexanone

\section{Introduction}

Heterocycles as the most prevalent organic compounds are present in various drugs, natural products, vitamins and bioactive compounds. ${ }^{1-3}$ They have been found to be useful as anti-HIV, ${ }^{4}$ anti-tumor, ${ }^{5,6}$ anti-inflammatory, ${ }^{7-9}$ anti-malarial, ${ }^{10,11}$ anti-depressant, anti-microbial, anti-bacterial and anti-fungal agents. ${ }^{12}$ Most heterocycles are used in materials science such as fluorescent sensors, dyes, data storage, plastics, illuminators and analytical reagents., ${ }^{3,13}$ Also, they are applied as important intermediates for the synthesis of medicinal compounds. ${ }^{14}$ Nitrogen-containing six-membered heterocycles, due to their high biological activity are of interest to both medicinal chemists and biochemists. ${ }^{15,16}$ Among them, pyridine and its derivatives play the most fundamental structural role in many natural compounds and medicinally beneficial molecules. ${ }^{17,18}$ Owing to the great variety of biologically active pyridines, it is not surprising that the pyridine ring system has become a vital basic component in many pharmaceutical agents. Some of the pyridine-derived drugs are trademarks of Reyataz and Gleevec drugs that are prescribed for HIV and chronic anemia, respectively. Some natural alkaloid products based on pyridine are nicotine and niacin derivatives. ${ }^{19}$ In particular, condensed pyridines are known for their several biological activities. For example, pyranopyridines have been proven to be the most active anti-tumor heterocyclic systems with activity against various filamentous tumors and specific activity against lung and ovarian cancer cells and antimicrobial action. $^{20,21}$ Moreover, pyrazolo-[3,4- $\left.b\right]$ pyridine derivatives are an important class of fused pyridines with a broad spectrum of biological activities that find widespread use in the pharmaceutical industries. ${ }^{22}$ Another class of fused pyridine derivatives is thiazolo[4,5-b]pyridin, which have anti-inflammatory and antimicrobial activity against human and veterinary pathogens. Also, some of these compounds have antifungal and anti-tumor activity. ${ }^{23,24}$

Coumarin-thiazole scaffolds are used as fluorescence probes for staining and imaging of DNA and to study the biological function of cell membranes. ${ }^{25,26}$ Other compounds fused to the coumarin ring, such as pyranochromenes as the most imperative fused polycyclic heterocycles have gained attention for their extensive occurrence in important pharmaceutical drugs. ${ }^{27}$ Many of pyranochromene derivatives exhibit significant biological activity, such as excellent antimicrobial potency, spasmolytic, anticoagulant, diuretic, cytotoxic, antituberculosis, anticancer, and antianaphylactic activities. ${ }^{28-30}$ Some of these compounds have found the use for the treatment of neurodegenerative diseases, including Parkinson's disease, Down's syndrome, Alzheimer's disease and AIDS associated dementia. ${ }^{31}$ Furthermore, pyranochromene derivatives have been applied as the key intermediates for the preparation of thioxo-imidazolidinedione, dithioxodiazetidine and Schiff's bases. ${ }^{32}$ 
Considering the above reports and in connection with our program on the synthesis of polycyclic compounds, ${ }^{33-40}$ we present in this paper an efficient and environmentally benign strategy for the synthesis of pyranochromene fused with pyridine derivatives. For this purpose, initially, pyrano[2,3-c]chromenes were prepared via the reaction between 4-hydroxycumarin (1), aromatic aldehydes 2 and malononitrile (3) using silica sodium carbonate (SSC) (Scheme 1). ${ }^{28}$ Subsequently, the synthesized pyrano[2,3-c]chromenes were reacted by dimethyl acetylenedicarboxylate (DMAD, 5) or cyclohexanone (6) to yield chromenopyrano[2,3-b]pyridine derivatives 7 and 8 (Scheme 2).

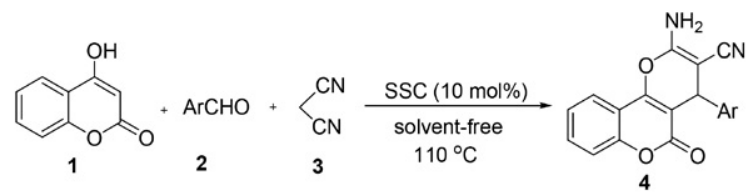

Scheme 1. SSC-catalyzed synthesis of pyranochromenes 4 .<smiles>N#CC1=C(N)Oc2c(c(=O)oc3ccccc23)C1Br</smiles>

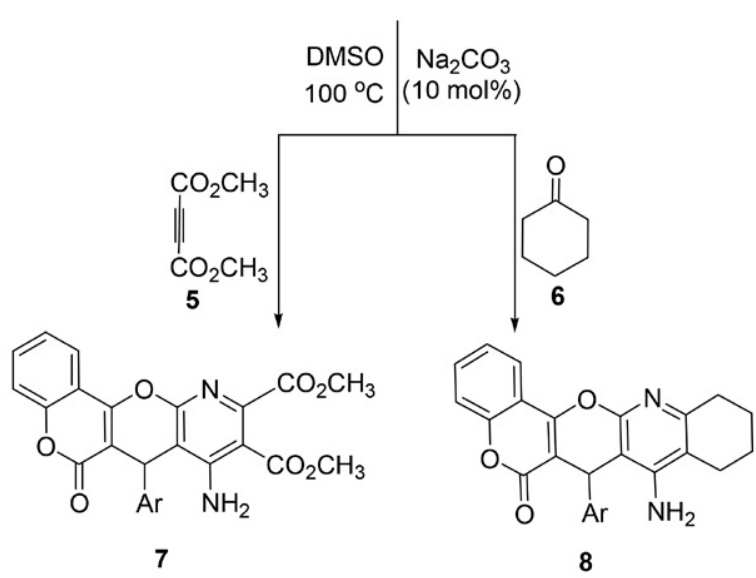

Scheme 2. Synthesis of chromenopyrano[2,3-b]pyridine derivatives 7 and $\mathbf{8}$ in the presence of $\mathrm{Na}_{2} \mathrm{CO}_{3}$ as the catalyst.

\section{Results and Discussion}

First, pyrano[2,3-c]chromenes 4 were prepared and identified according to the mentioned method (Table 1). In the following, in order to optimize the conditions, the reaction between pyranochromene $\mathbf{4 a}$ and DMAD was selected as a model system. The reaction was not completed in the absence of a catalyst at room temperature. Next, the model reaction was performed in the presence of $5 \mathrm{~mol} \%$ of Na${ }_{2} \mathrm{CO}_{3}$ in various solvents such as acetone, $\mathrm{CH}_{2} \mathrm{Cl}_{2}$, EtOAc, DMF and DMSO. As can be seen in Table 2, the best result was obtained by performing the reaction mixture in DMSO $\left(100{ }^{\circ} \mathrm{C}\right)$ to yield product $7 \mathbf{a}$. Next, we evaluated the required amount of the catalyst for this transformation. When $10 \mathrm{~mol} \%$ of $\mathrm{Na}_{2} \mathrm{CO}_{3}$ was used, the reaction efficiently proceeded and was complete in shorter reaction time. By further increasing the catalyst amount no appreciable improvement in the product yield and reaction time was observed. Various bases were screened for their efficiency in this reaction. We obtained the best yield of $7 \mathbf{a}$ when the reaction was performed by $\mathrm{Na}_{2} \mathrm{CO}_{3}$. Also, low temperatures led to the reaction product in a very low yield. In short, according to the obtained results, the best yield was achieved in DMSO at $100{ }^{\circ} \mathrm{C}$ in the presence of $10 \mathrm{~mol} \%$ of $\mathrm{Na}_{2} \mathrm{CO}_{3}$ (Table 2, Entry 8 ). In view of the success of the above reaction and having established the optimal conditions, we then investigated the scope and general applicability of this methodology by using different pyranochromenes and the results are given in Table 3. The structures of the synthesized compounds 7 were deduced from their elemental analysis, IR, ${ }^{1} \mathrm{H}$ and ${ }^{13} \mathrm{C}$ NMR spectroscopy. Subsequently, by employing this method, a number of chromenopyrano[2,3-b] pyridines 8 were produced $v i a$ the reaction between pyrano[2,3-c]chromenes 4 and cyclohexanone (6) under the optimized reaction conditions. In the majority of cases, the reactions performed cleanly and the desirable products $\mathbf{8}$ were formed in high

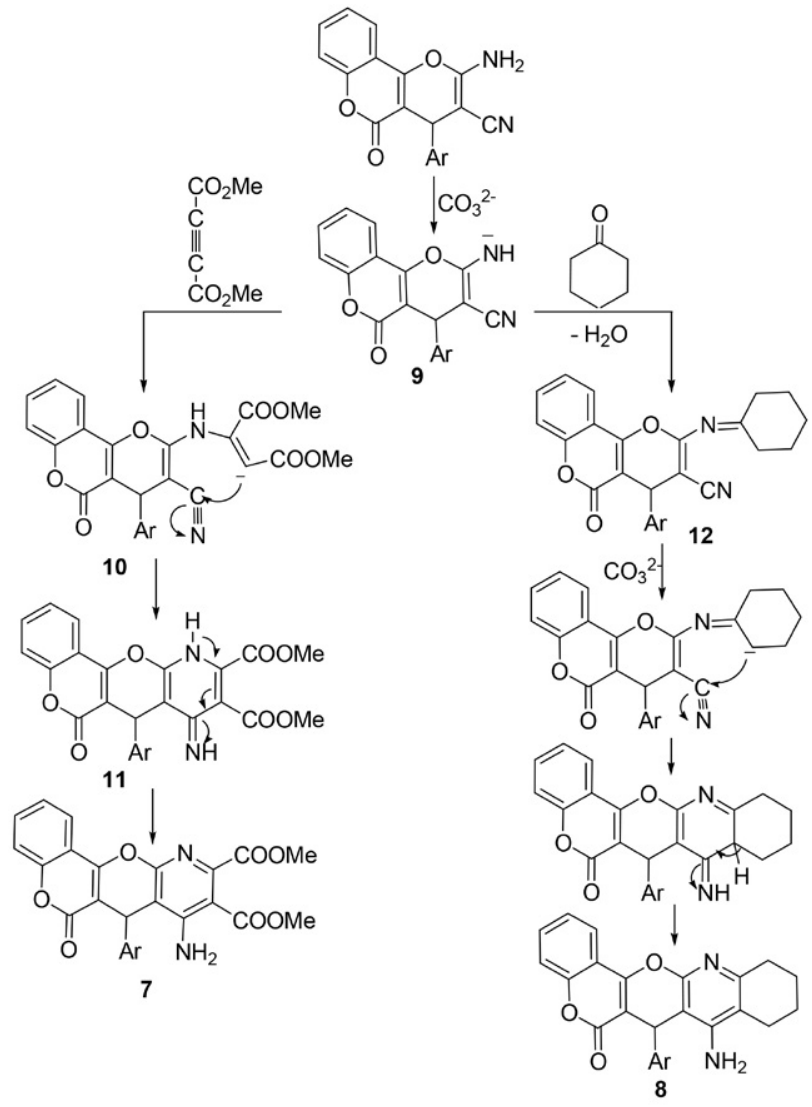

Scheme 3. Proposed mechanism for the synthesis of chromenopyrano[2,3-b]pyridines 7 and 8 using sodium carbonate. 
Table 1. Synthesis of pyrano[2,3-c] chromenes 4 using SSC catalyst.

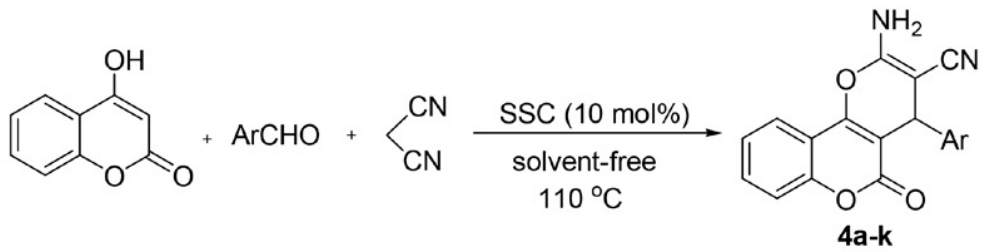

4a $\mathrm{C}_{6} \mathrm{H}_{5}$

${ }^{\mathrm{a}}$ Isolated yield.

Keshavarz et al.: Efficient and Facile Synthesis of Chromenopyrano[2,3-b] ... 
Table 2. Synthetic results of $7 \mathbf{a}$ under different reaction conditions.<smiles></smiles>

\begin{tabular}{|c|c|c|c|c|}
\hline Entry & Catalyst (mol \%) & Solvent/Temp. $\left({ }^{\circ} \mathrm{C}\right)$ & Time (h) & Yield $^{\mathrm{a}}(\%)$ \\
\hline 1 & None & None/25 & 24 & 5 \\
\hline 2 & $\mathrm{Na}_{2} \mathrm{CO}_{3}(5)$ & EtOAc/reflux & 5 & 40 \\
\hline 3 & $\mathrm{Na}_{2} \mathrm{CO}_{3}(5)$ & DMSO/100 & 5 & 50 \\
\hline 4 & $\mathrm{Na}_{2} \mathrm{CO}_{3}(5)$ & $\mathrm{CH}_{2} \mathrm{Cl}_{2} /$ reflux & 5 & 30 \\
\hline 5 & $\mathrm{Na}_{2} \mathrm{CO}_{3}(5)$ & Acetone/reflux & 5 & 40 \\
\hline 6 & $\mathrm{Na}_{2} \mathrm{CO}_{3}(5)$ & $\mathrm{DMF} / 100$ & 5 & 30 \\
\hline 7 & $\mathrm{Na}_{2} \mathrm{CO}_{3}(1)$ & DMSO/100 & 5 & 30 \\
\hline 8 & $\mathrm{Na}_{2} \mathrm{CO}_{3}(10)$ & DMSO/100 & 4 & 80 \\
\hline 9 & $\mathrm{Na}_{2} \mathrm{CO}_{3}(15)$ & DMSO/100 & 4 & 75 \\
\hline 10 & Piperidine (10) & DMSO/100 & 5 & 35 \\
\hline 11 & $\mathrm{NaOH}(10)$ & DMSO/100 & 5 & 42 \\
\hline 12 & $\mathrm{KOH}(10)$ & DMSO/100 & 5 & 45 \\
\hline 13 & $\mathrm{Na}_{2} \mathrm{CO}_{3}(10)$ & $\mathrm{DMSO} / 50$ & 4 & 37 \\
\hline 14 & $\mathrm{Na}_{2} \mathrm{CO}_{3}(10)$ & $\mathrm{DMSO} / 70$ & 4 & 50 \\
\hline
\end{tabular}

${ }^{\mathrm{a}}$ Isolated yield.

Table 3. Synthesis of chromenopyrano[2,3-b] pyridine derivatives 7 and $\mathbf{8}$ in the presence of $\mathrm{Na}_{2} \mathrm{CO}_{3}$ catalyst.<smiles>N#CC1=C(N)Oc2c(c(=O)oc3ccccc23)C1[10OH]</smiles>

\begin{tabular}{|c|c|c|c|c|c|}
\hline Entry & ry Ar & Product & Time (h) & Yield ${ }^{\mathrm{a}}(\%)$ & $\operatorname{Mp}$ [Ref.] $\left({ }^{\circ} \mathrm{C}\right)$ \\
\hline $7 \mathbf{a}$ & $\mathrm{C}_{6} \mathrm{H}_{5}$ & & 4 & 80 & decomp. $>300^{b}$ \\
\hline $7 \mathbf{b}$ & $4-\mathrm{Cl}-\mathrm{C}_{6} \mathrm{H}_{4}$ & & 3 & 75 & decomp. $>300^{\mathrm{b}}$ \\
\hline $7 \mathrm{c}$ & $4-\mathrm{Br}-\mathrm{C}_{6} \mathrm{H}_{4}$ & & 3 & 73 & $290-291^{b}$ \\
\hline
\end{tabular}

Keshavarz et al.: Efficient and Facile Synthesis of Chromenopyrano[2,3-b] ... 


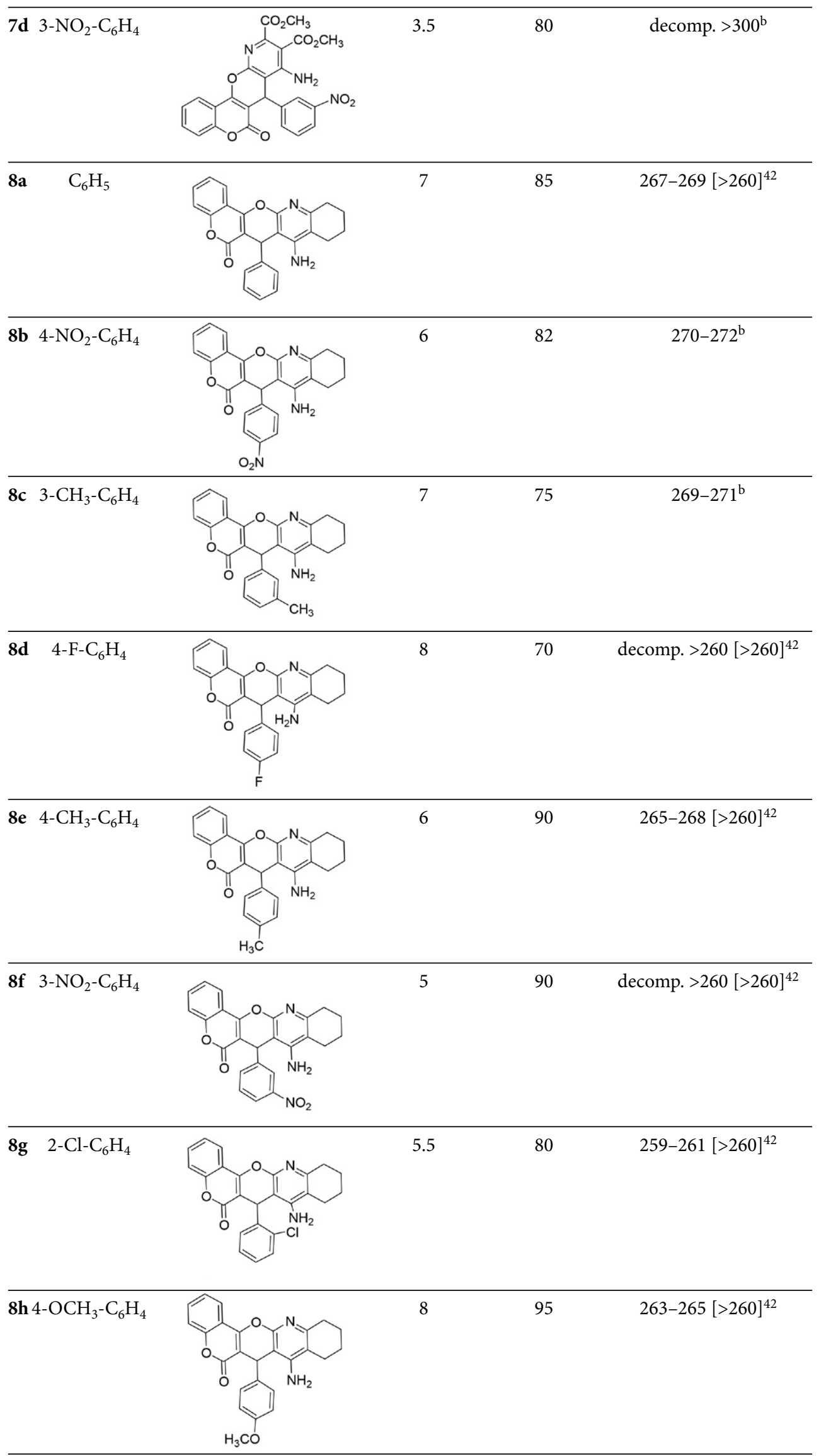

Keshavarz et al.: Efficient and Facile Synthesis of Chromenopyrano[2,3-b] ... 


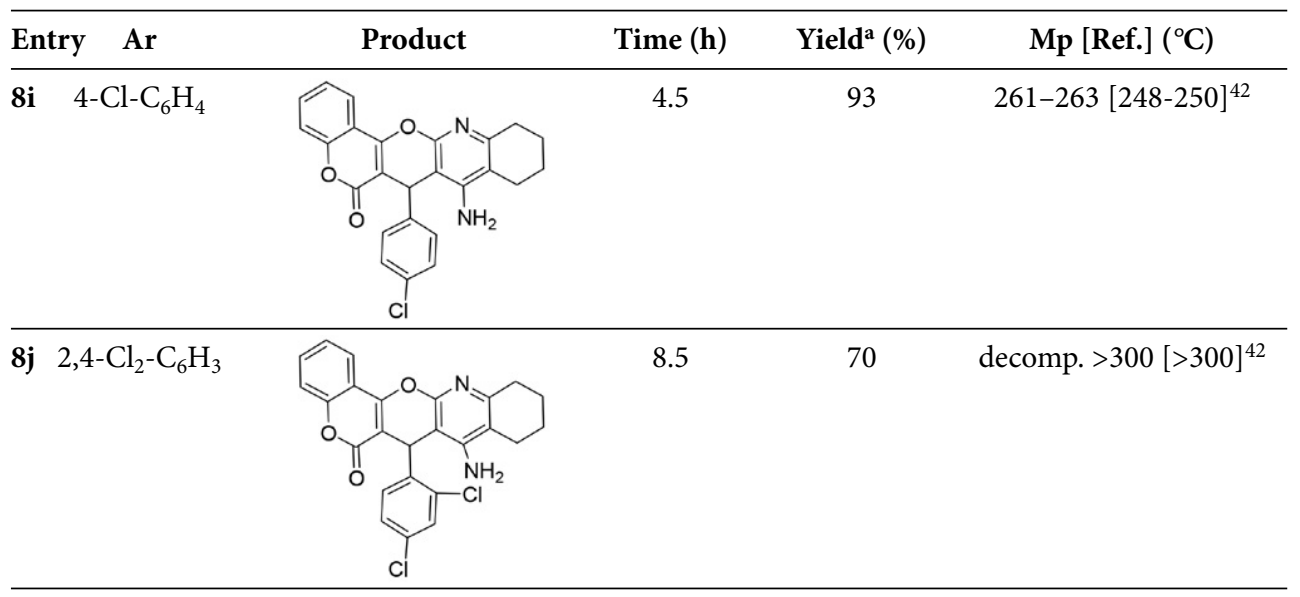

${ }^{\mathrm{a}}$ Isolated yield. ${ }^{\mathrm{b}}$ Novel compound.

yields and the nature of the Ar group appeared to have no notable effect on the reaction rate (Table 3 ).

We propose a mechanism for the $\mathrm{Na}_{2} \mathrm{CO}_{3}$-catalyzed synthesis of chromenopyrano[2,3-b]pyridine derivatives 7 (Scheme 3). Firstly, intermediate 9 is produced by the deprotonation of $\mathrm{NH}_{2}$ of pyranochromene in the presence of $\mathrm{Na}_{2} \mathrm{CO}_{3}$. Next, a Michael-type addition of $\mathrm{NH}^{-}$to dimethyl acetylenedicarboxylate creates adduct 10. The intramolecular cyclization of $\mathbf{1 0}$ gives adduct $\mathbf{1 1}$ which rearranges into the product 7 . The synthesis of product 8 also can be visualized as proceeding through a condensation of intermediate 9 with cyclohexanone to produce 12. Subsequently, cyclization and then tautomerization of $\mathbf{1 2}$ gives the desired product 8 (Scheme 3).

\section{Experimental}

All chemicals and reagents were purchased from Flu$\mathrm{ka}$ and Merck companies. The reaction progress was monitored by using TLC on silica gel polygram SIL G/UV254 plates. Reported melting points were determined by an electrothermal KSB1N apparatus. ${ }^{1} \mathrm{H}$ NMR spectra were recorded in DMSO- $d_{6}$ on a Bruker Avance Ultra Shield $400 \mathrm{MHz}$ instrument spectrometer and ${ }^{13} \mathrm{C}$ NMR spectra were recorded at $100 \mathrm{MHz}$. A Vario-El CHN instrument at the Isfahan Industrial University was used for the elemental analyses. IR spectra were obtained with a JASCO FT-IR/680 instrument spectrometer using $\mathrm{KBr}$ pellets.

\section{Procedure for the Preparation of the SSC Catalyst}

Silica sodium carbonate (SSC) was synthesized as a low-cost, recyclable and green catalyst in two steps according to the literature procedure. ${ }^{43}$ Initially, by adding thionyl chloride gradually to the silica gel, the silica chloride was prepared. Then, silica chloride and sodium bicarbonate were refluxed in hexane solvent for 24 hours. The solid product obtained, after washing and drying was used as a silica sodium carbonate catalyst.
General Procedure for the Synthesis of Pyranochromenes 4 SSC $(0.1 \mathrm{mmol}, 0.2 \mathrm{~g})$ was added to a mixture of malononitrile, aryl aldehyde, and 4-hydroxycoumarin at 110 ${ }^{\circ} \mathrm{C}$ under solvent-free conditions. The reaction progress was monitored by TLC. After completion of the reaction, boiling EtOAc $(10 \mathrm{~mL})$ was added, and the catalyst was separated by filtration. To further purifiy the product, obtained powder was recrystallized from $\mathrm{EtOH} .{ }^{28}$

\section{Preparation of Chromenopyrano[2,3-b]pyridines 7}

A solution of DMAD (1 mmol), compound 4 (1 $\mathrm{mmol})$ and $\mathrm{Na}_{2} \mathrm{CO}_{3}(10 \mathrm{~mol} \%)$ in DMSO $(5 \mathrm{~mL})$ was stirred at $100{ }^{\circ} \mathrm{C}$ for the appropriate time. The progress of the reaction was monitored by TLC (EtOAc/ $n$-hexan). After completion of the reaction, the catalyst was separated by filtration and the solvent was evaporated under reduced pressure. The obtained products were purified by column chromatography.

\section{Preparation of Chromenopyrano[2,3-b]pyridines 8}

A mixture of compound $4(1 \mathrm{mmol})$, cyclohexanone (5) (2 mmol) and $\mathrm{Na}_{2} \mathrm{CO}_{3}(10 \mathrm{~mol} \%)$ in DMSO $(5 \mathrm{~mL})$ was stirred and heated at $100{ }^{\circ} \mathrm{C}$ in a preheated oil bath for a required time. After termination of the reaction as demonstrated by TLC (EtOAc/ $n$-hexan), the catalyst was separated by filtration. The solvent was removed and the crude powder 8 was purified by crystallization from EtOAc.

Dimethyl 8-Amino-6-oxo-7-phenyl-6H,7H-chromeno $\left[3^{\prime}, 4^{\prime}: 5,6\right]$ pyrano $[2,3-b]$ pyridine-9,10-dicarboxylate (7a). Yield: $80 \%(0.35 \mathrm{~g})$, IR $(\mathrm{KBr})\left(v_{\max }, \mathrm{cm}^{-1}\right) 3446,2918$, $1650,1640,1420,1321 .{ }^{1} \mathrm{H}$ NMR $\left(400 \mathrm{MHz}, \mathrm{DMSO}-d_{6}\right)$ $\delta 8.15(\mathrm{~s}, 2 \mathrm{H}), 7.52-8.13(\mathrm{~m}, 3 \mathrm{H}), 7.42-7.50(\mathrm{~m}, 3 \mathrm{H})$, 7.24-7.34 (m, 3H), $5.21(\mathrm{~s}, 1 \mathrm{H}), 2.35(\mathrm{~s}, 3 \mathrm{H}), 2.24(\mathrm{~s}, 3 \mathrm{H})$. ${ }^{13} \mathrm{CNMR}\left(100 \mathrm{MHz}, \mathrm{DMSO}-d_{6}\right) \delta 160.16,152.78,151.24$, $142.01,132.81,128.59,124.63,122.37,117.23,113.48$, $100.22,100.16,77.35,77.24,77.04,76.72,53.22,34.95$, 30.98. Anal. Calcd for $\mathrm{C}_{25} \mathrm{H}_{18} \mathrm{~N}_{2} \mathrm{O}_{7} \mathrm{C}, 65.50 ; \mathrm{H}, 3.96 ; \mathrm{N}$, 6.11. Found C, 65.53; H, 3.90; N, 6.18 . 
Dimethyl 8-Amino-7-(4-chlorophenyl)-6-oxo-6 $\mathrm{H}, 7 \mathrm{H}$ chromeno $\left[3^{\prime}, 4^{\prime}: 5,6\right]$ pyrano[2,3-b] pyridine-9,10-dicarboxylate (7b). Yield: $75 \%(0.37 \mathrm{~g}), \mathrm{IR}(\mathrm{KBr})\left(v_{\max }, \mathrm{cm}^{-1}\right)$ 3417, 2946, 1648, 1636, 1490, 1321, 777. ${ }^{1} \mathrm{H}$ NMR (400 $\left.\mathrm{MHz}, \mathrm{DMSO}-d_{6}\right) \delta 7.84(\mathrm{~s}, 2 \mathrm{H}), 7.71(\mathrm{~s}, 2 \mathrm{H}), 7.11-7.52$ (m, 5H), 7.09 (s, 1H), 5.45 (s, 1H), 3.35 (s, 3H), 2.51-2.53 $(\mathrm{m}, 3 \mathrm{H}) .{ }^{13} \mathrm{C}$ NMR $\left(100 \mathrm{MHz}, \mathrm{DMSO}-d_{6}\right) \delta 169.90,168.01$, $165.61,154.02,153.91,145.04,142.75,140.74,138.25$, 137.42, 131.91, 125.07, 122.51, 119.51, 112.17, 97.94, 79.88, 49.07, 31.15. Anal. Calcd for $\mathrm{C}_{25} \mathrm{H}_{17} \mathrm{ClN}_{2} \mathrm{O}_{7} \mathrm{C}, 60.92 ; \mathrm{H}$, 3.48; N, 5.68. Found C, 60.91; H, 3.51; N, 5.65.

Dimethyl 8-Amino-7-(4-bromophenyl)-6-oxo-6H,7Hchromeno $\left[3^{\prime}, 4^{\prime}: 5,6\right]$ pyrano $[2,3-b]$ pyridine-9,10-dicarboxylate $(7 \mathrm{c})$. Yield: $73 \%(0.39 \mathrm{~g}), \mathrm{IR}(\mathrm{KBr})\left(v_{\max }, \mathrm{cm}^{-1}\right)$ 3450, 2923, 1667, 1563, 1488, 1348, 764. ${ }^{1} \mathrm{H}$ NMR (400 $\left.\mathrm{MHz}, \mathrm{DMSO}-d_{6}\right) \delta 7.941(\mathrm{~s}, 2 \mathrm{H}), 7.588-7.918(\mathrm{~m}, 3 \mathrm{H})$, $7.314-7.438(\mathrm{~m}, 2 \mathrm{H}), 7.147(\mathrm{~d}, J=8.1 \mathrm{~Hz}, 3 \mathrm{H}), 6.332(\mathrm{~s}$, $1 \mathrm{H}), 2.762(\mathrm{~s}, 3 \mathrm{H}), 2.523(\mathrm{~s}, 3 \mathrm{H}) .{ }^{13} \mathrm{C} \mathrm{NMR}(100 \mathrm{MHz}$, DMSO- $\left.d_{6}\right) \delta 168.24,166.04,165.14,161.19,152.74$, $150.21,144.47,140.36,132.38,131.31,129.61,124.42$, 124.17, 118.99, 118.51, 116.42, 104.27, 97.91, 94.51, 52.12, 36.24. Anal. Calcd for $\mathrm{C}_{25} \mathrm{H}_{17} \mathrm{BrN}_{2} \mathrm{O}_{7} \mathrm{C}, 55.88 ; \mathrm{H}, 3.19 ; \mathrm{N}$, 5.21. Found C, 55.90; H, 3.15; N, 5.27.

Dimethyl 8-Amino-7-(3-nitrophenyl)-6-oxo-6H,7Hchromeno $\left[3^{\prime}, 4^{\prime}: 5,6\right]$ pyrano[2,3-b]pyridine-9,10-dicarboxylate (7d). Yield: $80 \%(0.40 \mathrm{~g})$, IR $(\mathrm{KBr})\left(v_{\max }, \mathrm{cm}^{-1}\right)$ $3415,2953,1634,1554,1530,1437,1349 .{ }^{1} \mathrm{H}$ NMR $(400$ MHz, DMSO- $\left.d_{6}\right) \delta 8.033(\mathrm{~d}, J=7.8 \mathrm{~Hz}, 2 \mathrm{H}), 7.937(\mathrm{~s}, 1 \mathrm{H})$, $7.875(\mathrm{~d}, J=7.5 \mathrm{~Hz}, 1 \mathrm{H}), 7.503-7.645(\mathrm{~m}, 4 \mathrm{H}), 7.267-7.352$ $(\mathrm{m}, 2 \mathrm{H}), 6.404(\mathrm{~s}, 1 \mathrm{H}), 3.581(\mathrm{~s}, 3 \mathrm{H}), 2.564(\mathrm{~s}, 3 \mathrm{H}) .{ }^{13} \mathrm{C}$ NMR $\left(100 \mathrm{MHz}, \mathrm{DMSO}-d_{6}\right) \delta 49.07,50.46,82.67,102.51$, $106.85,110.73,112.09,126.68,128.51,129.39,132.14$, $138.14,139.24,144.53,154.53,157.15,160.46,161.82$, 167.32, 167.86. Anal. Calcd for $\mathrm{C}_{25} \mathrm{H}_{17} \mathrm{~N}_{3} \mathrm{O}_{9} \mathrm{C}, 59.65 ; \mathrm{H}$, $3.40 ; \mathrm{N}, 8.35$. Found C, 59.66; H, 3.43; N, 8.32.

8-Amino-7-phenyl-9,10,11,12-tetrahydro-7 H-5,14-dioxa-13-azabenzo[ $[\boldsymbol{a}]$ naphthacen-6-one $(8 \mathrm{a})$. Yield: $85 \%$ $(0.34 \mathrm{~g})$, IR (KBr) $\left(v_{\max }, \mathrm{cm}^{-1}\right) 3419,2933,1727,1670$, $1641,1602,1492,1454,1382,1307,1201,1039,754,700$, $543,460 .{ }^{1} \mathrm{H}$ NMR $\left(400 \mathrm{MHz}, \mathrm{DMSO}-d_{6}\right) \delta 8.05(\mathrm{~d}, J=8.0$ $\mathrm{Hz}, 1 \mathrm{H}), 8.01(\mathrm{t}, J=7.8 \mathrm{~Hz}, 1 \mathrm{H}), 7.77(\mathrm{~d}, J=8.0 \mathrm{~Hz}, 1 \mathrm{H})$, $7.55(\mathrm{t}, J=8.0 \mathrm{~Hz}, 1 \mathrm{H}), 7.52(\mathrm{~d}, J=7.8 \mathrm{~Hz}, 1 \mathrm{H}), 7.31(\mathrm{~s}$, $2 \mathrm{H}), 7.26(\mathrm{~d}, J=8.0 \mathrm{~Hz}, 3 \mathrm{H}), 7.24(\mathrm{~d}, J=8.0 \mathrm{~Hz}, 1 \mathrm{H}), 5.39$ (s, 1H), 2.81-2.90 (m, 2H), 1.77-1.95 (m, 6H). ${ }^{13} \mathrm{C}$ NMR $\left(100 \mathrm{MHz}, \mathrm{DMSO}-d_{6}\right) \delta 160.1,156.1,153.9,152.5,149.9$, $141.3,133.7,129.1,128.8,127.9,125.4,122.9,117.1,114.5$, 113.4, 106.1, 98.8, 40.6, 40.2, 39.3, 33.7, 21.3, 21.1. Anal. Calcd for $\mathrm{C}_{25} \mathrm{H}_{20} \mathrm{~N}_{2} \mathrm{O}_{3} \mathrm{C}, 75.74 ; \mathrm{H}, 5.08 ; \mathrm{N}, 7.07$. Found $\mathrm{C}$, 75.72; H, 5.10; N, 7.05.

8-Amino-7-(4-nitrophenyl)-9,10,11,12-tetrahydro-7H-5,14-dioxa-13-azabenzo $[\boldsymbol{a}]$ naphthacen-6-one (8b). Yield: $82 \%$ (0.36 g), IR (KBr) $\left(v_{\max }, \mathrm{cm}^{-1}\right) 3338,3230$, 2935, 2861, 1722, 1668, 1604, 1517, 1454, 1382, 1346,
1276, 1201, 1106, 1043, 906, 763, 543. ${ }^{1} \mathrm{H}$ NMR (400 MHz, DMSO $\left.-d_{6}\right) \delta 8.16(\mathrm{~d}, J=8.0 \mathrm{~Hz}, 2 \mathrm{H}), 8.04(\mathrm{~d}, J=8.0 \mathrm{~Hz}$, $1 \mathrm{H}), 7.79(\mathrm{~d}, J=8.0 \mathrm{~Hz}, 2 \mathrm{H}), 7.55(\mathrm{~s}, 2 \mathrm{H}), 7.52(\mathrm{t}, J=8.0$ $\mathrm{Hz}, 3 \mathrm{H}), 5.54(\mathrm{~s}, 1 \mathrm{H}), 2.78-2.93(\mathrm{~m}, 2 \mathrm{H}), 1.77-1.88(\mathrm{~m}$, $6 \mathrm{H}) .{ }^{13} \mathrm{C}$ NMR $\left(100 \mathrm{MHz}, \mathrm{DMSO}-d_{6}\right) \delta 160.0,156.2,153.8$, $149.7,141.1,137.9,133.7,129.6,128.6,126.2,122.9,117.1$, 114.5, 106.1, 98.9, 40.6, 40.4, 40.2, 40.0, 33.7, 22.8, 21.5, 21.3. Anal. Calcd for $\mathrm{C}_{25} \mathrm{H}_{19} \mathrm{~N}_{3} \mathrm{O}_{5} \mathrm{C}, 68.02 ; \mathrm{H}, 4.34 ; \mathrm{N}$, 9.52. Found C, 68.06; $\mathrm{H}, 4.37 ; \mathrm{N}, 9.50$.

8-Amino-7-(3-metylphenyl)-9,10,11,12-tetrahydro7H-5,14-dioxa-13-azabenzo[ $[a]$ naphthacen-6-one (8c). Yield: $75 \%(0.31 \mathrm{~g})$, IR (KBr) $\left(v_{\max }, \mathrm{cm}^{-1}\right) 3334,3232$, 2937, 1727, 1668, 1641, 1604, 1490, 1454, 1382, 1307, 1274, $1201,1043,902,763,586 .{ }^{1} \mathrm{H}$ NMR $\left(400 \mathrm{MHz}, \mathrm{DMSO}-d_{6}\right)$ $\delta 8.01(\mathrm{~d}, J=8.0 \mathrm{~Hz}, 1 \mathrm{H}), 7.74(\mathrm{t}, J=8.0 \mathrm{~Hz}, 2 \mathrm{H}), 7.53(\mathrm{t}$, $J=8.0 \mathrm{~Hz}, 2 \mathrm{H}), 7.47(\mathrm{~d}, J=8 \mathrm{~Hz}, 1 \mathrm{H}), 7.35(\mathrm{~s}, 2 \mathrm{H}), 7.17$ (t, $J=8.0 \mathrm{~Hz}, 1 \mathrm{H}), 7.03(\mathrm{~d}, J=8.0 \mathrm{~Hz}, 1 \mathrm{H}), 5.34(\mathrm{~s}, 1 \mathrm{H})$, 2.83-2.98 (m, 2H), $2.52(\mathrm{~s}, 3 \mathrm{H}), 1.77-1.90(\mathrm{~m}, 6 \mathrm{H}) .{ }^{13} \mathrm{C}$ NMR $\left(100 \mathrm{MHz}, \mathrm{DMSO}-d_{6}\right) \delta 159.9,156.5,153.6,152.5$, $149.4,146.8,141.0,137.9,133.7,129.6,128.7,126.3,125.4$, 122.9, 117.1, 114.5, 113.2, 106.1, 98.9, 40.1, 39.3, 33.6, 22.8, 21.5, 21.2, 21.0. Anal. Calcd for $\mathrm{C}_{26} \mathrm{H}_{22} \mathrm{~N}_{2} \mathrm{O}_{3} \mathrm{C}, 76.08 ; \mathrm{H}$, $5.40 ; \mathrm{N}, 6.82$. Found C, 76.05; H, 5.36; N, 6.78.

\section{Conclusions}

In summary, we have demonstrated that the reaction between pyrano[2,3-c]chromenes and dimethyl acetylenedicarboxylate or cyclohexanone in the presence of sodium carbonate provides a simple method for the preparation of chromenopyrano[2,3-b]pyridine derivatives. One of the important benefits of this protocol is the use of sodium carbonate as a commercially available and inexpensive catalyst. This study has helped to find an effective production of biologically useful compounds belonging to the family of pyranopyridines.

\section{Acknowledgements}

The authors gratefully acknowledge the partial support of this work by Yasouj University, Iran.

\section{References}

1. H. M. Elwahy, M. R. Shaaban, RSC Adv. 2015, 5, 7565975710.

2. X. F. Wu, H. Neumann, M. Beller, Chem. Rev. 2013, 113, 1-35. DOI:10.1039/C5RA11421G

3. B. Jiang, T. Rajale, W. Wever, S. J. Tu, G. Li, Chem. Asian J. 2010, 5, 2318-2335. DOI:10.1002/asia.201000310

4. E. G. Hammam, N. A. A. El-hafeza, W. H. Midurab, M. Z. Mikołajczyk, Naturforsch. B: Chem. Sci. 2000, 55, 417-423. DOI:10.1515/znb-2000-0511 
5. K. Ishiguro, K. Takahashi, K. Yazawa, S. Sakiyama, T. Arai, J. Biol. Chem. 1981, 256, 2162-2167.

6. R. M. Mohareb, N. N. E. El-Sayed, M. E. Abdelaziz Molecules 2012, 17, 8449-8463. DOI:10.3390/molecules 17078449

7. G. E. Amr, M. M. Abdulla, Bioorg. Med. Chem. 2006, 14, 4341-4352. DOI:10.1016/j.bmc.2006.02.045

8. M. Ghate, R. A. Kusanur, M. V. Kulkarni, Eur. J. Med. Chem. 2005, 40, 882-887. DOI:10.1016/j.ejmech.2005.03.025

9. S. Shaf, M. M. Alam, N. Mulakayala, C. Mulakayala, G. Vanaja, A. M. Kalle, R. Pallu, M. S. Alam, Eur. J. Med. Chem. 2012, 49, 324-333. DOI:10.1016/j.ejmech.2012.01.032

10. M. V. B. Reddy, C. R. Su, W. F. Chiou, Y. N. Liu, R. Y. H. Chen, K. F. Bastow, K. H. Lee, T. S. Wu, Bioorg. Med. Chem. 2008, 16, 7358-7370. DOI:10.1016/j.bmc.2008.06.018

11. V. V. Shinde, M. V. Reddy, Y. H. Kim, B. K. Cho, Y. T. Jeong, Monatsh. Chem. 2015, 146, 673-682.

DOI:10.1007/s00706-014-1380-9

12. M. Yusuf, P. Jain, Arab. J. Chem. 2011, 5, 553-596.

13. M. A. P. Martins, C. P. Frizzo, D. N. Moreira, L. Buriol, P. Machado, Chem. Rev. 2009, 109, 4140-4182.

DOI:10.1021/cr9001098

14. M. A. P. Martins, C. P. Frizzo, D. N. Moreira, N. Zanatta, H. G. Bonacorso, Chem. Rev. 2008, 108, 2015-2050.

DOI:10.1021/cr078399y

15. L. Zheng, J. Ju, Y. Bin, R. Hua, J. Org. Chem. 2012, 77, 57945800. DOI:10.1021/jo3010414

16. S. A. Raw, C. D. Wilfred, R. J. K. Taylor, Org. Biomol. Chem. 2004, 2, 788-796. DOI:10.1039/b315689c

17. M. Movassaghi, M. D. Hill, O. K. Ahmad, J. Am. Chem. Soc. 2007, 129, 10096-10097. DOI:10.1021/ja073912a

18. A. Antonyraj, S. Kannan, Appl. Catal. A 2008, 338, 121-129. DOI:10.1016/j.apcata.2007.12.028

19. M. D. Hill, Chem. Eur. J. 2010, 16, 12052-12062.

DOI: $10.1002 /$ chem. 201001100

20. A. F. S. Rostom, G. S. Hassan, H. I. El-Subbagh, Arch. Pharm. Chem. Life Sci. 2009, 342, 584-586.

DOI:10.1002/ardp.200900062

21. M. M. Ghorabaa, I. Y. Hassan, Phosph. Sulfur Silicon Relat. Elem. 1998, 141,251-261. DOI:10.1080/10426509808033737

22. Z. Huang, Y. Hu, Y. Zhou, D. Shi, ACS Comb. Sci. 2011, 12, 45-49. DOI:10.1021/co1000162

23. T. I. Chaban, V. V. Ogurtsov, V. S. Matiychuk, I. G. Chaban, I. L. Demchuk, I. A. Nektegayev, Acta Chim. Slov. 2019, 66, 103-111. DOI:10.17344/acsi.2018.4570

24. T. I. Chaban, R. R. Panchuk, O. V. Klenina, N. R. Skorokhyd, V. V. Ogurtsov, I. G. Chaban, Biopolym. Cell. 2012, 28, 389396. DOI: $10.7124 /$ bc. 000075
25. S. Pajk, M. Garvas, J. Štrancar, Acta Chim. Slov. 2019, 66, 668674. DOI:10.17344/acsi.2019.5089

26. N. Narayanaswamy, M. Kumar, S. Das, R. Sharma, P. K. Samanta, S. K. Pati, S. K. Dhar, T. K. Kundu, T. Govindaraju, Sci. Rep. 2014, 25, 6476-6483.

27. H. Mohamadi Tanuraghaj, M. Farahi, Tetrahedron Lett. 2019, 60, 557-559. DOI:10.1016/j.tetlet.2019.01.030

28. M. Farahi, M. Abdipour, Org. Chem. Res. 2018, 4, 182-193.

29. C. Mungra, M. P. Patel, D. P. Rajani, R. G. Patel, Eur. J. Med. Chem. 2011, 46, 4192-4200.

DOI:10.1016/j.ejmech.2011.06.022

30. B. D. Parmar, T. R. Sutariya, G. C. Brahmbhatt, N. J. Parmar, R. Kant, V. K. Gupta, J. Org. Chem. 2016, 81, 4955-4964. DOI:10.1021/acs.joc.6b00107

31. B. Sameem, M. Saeedi, M. Mahdavi, H. Nadri, F. Homayouni Moghadam, N. Edraki, M. I. Khan, M. Amini, Bioorg. Med. Chem. 2017, 25, 3980-3988. DOI:10.1016/j.bmc.2017.05.043

32. Z. M. Nofal, H. H. Fahmy, M. M. Kamel, A. I. Sarhan, A. S. Maghraby, Egypt. J. Chem. 2004, 47, 345-368.

33. H. Mohamadi Tanuraghaj, M. Farahi, Tetrahedron Lett. 2019 , 60,557-559. DOI:10.1016/j.tetlet.2019.01.030

34. M. Farahi, B. Karami, H. Mohamadi Tanuraghaj, Z. Bazrafshan, J. Heterocycl. Chem. 2018, 55, 125-131.

DOI:10.1002/jhet.3014

35. M. Farahi, B. Karami, H. Mohamadi Tanuraghaj, Tetrahedron Lett. 2015, 56, 1833-1836. DOI:10.1016/j.tetlet.2015.02.087

36. M. Farahi, B. Karami, Z. Banaki, F. Rastgoo, K. Eskandari, Monatsh. Chem. 2017, 148, 1469-1475.

DOI:10.1007/s00706-017-1932-X

37. M. Farahi, B. Karami, A. Jokar, K. Eskandari, Org. Prep. Proc. Int. 2017, 49, 514-524.

DOI:10.1080/00304948.2017.1380495

38. M. Farahi, M. Davoodi, M. Tahmasebi, Tetrahedron Lett. 2016, 57, 1582-1584. DOI:10.1016/j.tetlet.2016.02.101

39. M. Farahi, F. Tamaddon, B. Karami, S. Pasdar, Tetrahedron Lett. 2015, 56, 1887-1890. DOI:10.1016/j.tetlet.2015.02.105

40. H. Mohamadi Tanuraghaj, M. Farahi, RSC Adv. 2018, 8, 27818-27824. DOI:10.1039/C8RA05501G

41. H. Mehrabi, H. Abusaidi, J. Iran. Chem. Soc. 2010, 7, 890-894 DOI:10.1007/BF03246084

42. M. Khoobi, M. Alipour, A. Moradi, A. Sakhteman, H. Nadri, S. F. Razavi, M. Ghandi, A. Foroumadi, A. Shafiee, Eur. J. Med. Chem. 2013, 68, 291-300.

DOI:10.1016/j.ejmech.2013.07.045

43. K. Eskandari, B. Karami, S. Khodabakhshi, Catal. Commun. 2014, 54, 124-130. DOI:10.1016/j.catcom.2014.05.029 


\section{Povzetek}

$\mathrm{V}$ okviru te raziskave smo pripravili serijo novih in nekaj že znanih kromenopirano[2,3-b]piridinskih derivatov. Skladno z že objavljenimi postopki smo najprej z reakcijo med 4-hidroksikumarinom, aromatskimi aldehidi in malononitrilom ob prisotnosti silika natrijevega karbonata (SSC) kot katalizatorja pripravili pirano[2,3-c]kromenske derivate, ki smo jih $\mathrm{v}$ naslednji stopnji reagirali $\mathrm{z}$ dimetil acetilendikarboksilatom (DMAD) ali cikloheksanonom $\mathrm{v}$ prisotnosti natrijevega karbonata. Tako smo pripravili serijo kromenopirano[2,3-b]piridinskih derivatov. Za izvedbo našega protokola ne potrebujemo dragih katalizatorjev in vendar lahko potencialno bioaktivne heterociklične spojine pripravimo $\mathrm{z}$ odličnimi do visokimi izkoristki. 